\title{
Efficient Distributed Localization Algorithm for Ownership Identification of Reindeer Calf Using Wireless Sensor Networks
}

\author{
Gemma Morral $^{1 \star}$, Mohamad Y. Mustafa ${ }^{1 \star}$, Ndeye Amy Diengy ${ }^{2}$ \\ ${ }^{1}$ Department of Electrotechnology Faculty of Engineer Science and Technology, Tromsø University, 8505 Narvik, Norway \\ ${ }^{2} y I n s t i t u t$ Mines-Télécom, Télécom Bretagne - Université Européenne de Bretagne, Brest, France
}

\section{Abstract}

\section{Publication History:}

This paper presents a distributed algorithm for identifying the ownership of new-born reindeer calves using wireless sensor networks (WSNs). Reindeer are semi wild animals giving birth while in the wild. Although reindeer cows usually carry identification tags or signs of their owners, it is difficult to identify the ownership of the calves within a mixed herd. Currently, identification is performed in the traditional way which is stressful on both animals and herders and quite costly and time consuming. In this work, a localization algorithm implemented on a WSN is considered to get rid of matching mother reindeer to their calves in a fully automatic way. In particular, the proposed distributed localization algorithm is based on two phases where sensors nodes are attached to the reindeer in a confined space with the objective to identify the animals keeping close together the most of the time, which are reckoned to be the mother reindeer and her calf. At first, the initial estimated nodes' positions are obtained from

Received: March 10, 2017

Accepted: August 20, 2017

Published: August 22, 2017

\section{Keywords:}

Wireless Sensor Networks, Animal Localization, Reindeer Herding, Distributed Algorithm, Position Estimation an existing biased-maximum likelihood estimator (B-MLE) based on the log-normal shadowing model (LNSM). Secondly, each sensor node seeks to estimate its local map (i.e., its own position and those from its neighbors) by using an on-line distributed stochastic approximation algorithm (DSA) such the identification mother-calves can be done. We present numerical are issued to the standard ZigBee IEEE 802.15.4 operating at 2:4 GHz by means of radio devices WSN430 (equipped with CC2420 chips).

\section{Introduction}

\section{Context: ownership identification of reindeer calf}

Reindeer herding is the major economic activity of the Sami people, who are the indigenous people of northern Sweden, Norway and Finland. The Sami people lived and worked in reindeer herding groups, which consisted of different families within a designated area and which were formed of working partnerships, where members had individual rights to resources, but helped each other with the management of the herds, or when hunting and fishing. Reindeer husbandry in Norway today is a small industry on a national scale, but is important economically and in employment terms; it is also one of the most important parts of the Sami culture. Reindeer herding in Norway was regulated in 2007 and allowed only those who have the right to a reindeer earmark to undertake reindeer husbandry in the Sami reindeer herding area. This condition applied only to persons who are Sami themselves, whose parents or their grandparents have or had reindeer herding as their primary occupation. A reindeer earmark is a combination of one to many cuts in a reindeer's ears, which all together tell who the reindeer owner is. There are around 20 different approved cuts in addition to some 30 different combinations of cuts, which have their own names. A committee is in charge of approving earmarks before they are implemented and all reindeer in the Sami reindeer husbandry area shall be marked with the owner's registered earmark by 31 October the same year as it is born. Reindeer are semi-wild and require large areas for their grazing, they are also often frightened and are forced to flee from natural pastures. Most of the time, they are left to wander freely unaccompanied with their herders [1]. Reindeer herders typically make two migrations with their animals each year. During winter, reindeer are left to breed in the highlands, but just before spring, when food becomes scarcer in the mountains, natural instinct directs the herd to migrate to the low grounds for greener pastures [2]. Reindeer are intercepted by their herders and are taken to the spring pastures before the snow melts and before the mothers start calving. In spring, they move back to the mountainous coastal region where they feed throughout the summer, the calves are soon born and stay with their mothers for protection [3]. In autumn, the herders return to the grazing locations to gather the reindeer from the mountains before the breeding season starts. This is the time when the new calves are branded. Each calf is captured, vaccinated and is given an identification tag or ear mark to distinguish which cow the calf belongs to and to which owner they belong [2]. As meat production became more important since the beginning of the 20th century, reindeer herding became more extensive and Sami reindeer herders started to implement modern technologies, such as snow mobiles and various other mechanical and electronic aids, which became a major feature of modern reindeer herding. Among those technologies, ear identification tags are being used extensively. However, identifying the ownership of new born calves remains a major problem for reindeer herders [1]. Recognizing and matching reindeer calves to the different mothers is traditionally performed in two steps; first, the animals are gathered in a small pen. All female reindeer get individual numbers sprayed on the skin at each side of the animal. Each owner uses one specific color. Unique number plates are hanged to collars around the necks of the calves. Thereafter, the animals are released to larger pens to calm down and are put under surveillance by reindeer owners and herders using "Corresponding Author: Prof. Gemma Morral, Department of Electrotechnology Faculty of Engineer Science and Technology, Troms $\varnothing$ University (UiT The Arctic University of Norway), 8505 Narvik, Norway; E-mail: gemma.m.adell@uit.no

"Corresponding Author: Prof. Mohamad Y. Mustafa, Department of Electrotechnology Faculty of Engineer Science and Technology, Troms $\varnothing$ University (UiT The Arctic University of Norway), 8505 Narvik, Norway; E-mail: mohamad.y.mustafa@uit.no

Citation: Morral G, Mohamad Y, Diengy NA (2017) Efficient Distributed Localization Algorithm for Ownership Identification of Reindeer Calf Using Wireless Sensor Networks. Int J Comput Softw Eng 2: 120. doi: https://doi. org/10.15344/2456-4451/2017/120

Copyright: (C) 2017 Morral, et al. This is an open-access article distributed under the terms of the Creative Commons Attribution License, which permits unrestricted use, distribution, and reproduction in any medium, provided the original author and source are credited. 
Citation: Morral G, Mohamad Y, Diengy NA (2017) Efficient Distributed Localization Algorithm for Ownership Identification of Reindeer Calf Using Wireless Sensor Networks. Int J Comput Softw Eng 2: 120. doi: https://doi.org/10.15344/2456-4451/2017/120

Page 2 of 7

binoculars, in order to identify which reindeer calf is following which cow. Thereafter, reindeer owners compare the different observations to guaranty that the correct mother is matched to her calf [4]. The next step is to gather the reindeer herd again in a small pen, capture the calves, remove the number plates and replace them with the approved ear cutting or a tag. This handling procedure is a painstaking process, which demands considerable amount of man power, and long periods of observation of the herd, and is stressful to both herders and animals alike [4]. With the advancement of wireless communications and the wide spread of wireless networks, it has become possible to utilize those technologies for the benefits of reindeer herders and their animals. This paper proposes a technique based on WSN technology for ownership identification of new-born reindeer calves. Various prospective identification technologies such as RFID tag, GPS collars or UHF nodes and gateways are discussed in this work to explore their feasibility for the proposed application [1]. In this concept, it is proposed to equip the reindeer cows and calves with transmitter devices of the selected technology, and monitor the movement of the herd in a confined space, facilitated with a grid of signal receivers, corresponding to the transmitter device attached to the reindeer. A suitable algorithm has to be devised to analyze the gathered data and recognize the pairs of tags which keep close together most of the time, which are reckoned to be the mother reindeer and her calf.

\section{Overview of WSN-based localization techniques}

The problem of self-localization involving low-cost radio devices in wireless sensor networks (WSN) can be viewed as an example of the Internet of Things (IoT). Among the several applications related to this problem, location services may be namely offered by small devices carried by persons in indoor environments. Over the last ten years, several overview papers dealing with the description and classification of localization techniques in this context have been published (see, for instance, [5-7]).

In this work, we focus on the ranging technique relying on received signal strength indicator (RSSI) for indoor scenarios. In [8], the RSSI metric has been related to the Euclidean distance through a lognormal shadowing model (LNSM) describing the signal propagation in free-space. A first approach consists of assuming the LNSM and applying a classical maximum likelihood estimator (MLE) to compute the nodes' positions. This has been proposed, for instance, in [9], where the parameters of the propagation model are considered equal for all landmarks (i.e., nodes whose positions are known, also called anchor nodes). Following the same approach, the parameters have been considered different for each landmark in in [10]. The experimental results there obtained on real test beds show a mean localization accuracy of $1 \mathrm{~m}$.

When dealing with closed and relatively small spaces, RSSI is not accurate enough and the effects of multipath, possible blocking objects and antenna orientation may be also included in the propagation model as outliers (see, for instance, [11,12]). A biased-maximum likelihood estimator (B-MLE) involving a random factor related to the possibly outliers has been studied in [13], this estimator has been experimentally proved to reduce the mean error of the classical MLE. The optimization problem is defined for each single unknown position given several RSSI values from a set of surrounding landmarks.

Our work aims to solve the localization problem of a set of nodes' positions in a distributed and cooperative way, i.e., both landmarks and neighboring nodes communicate and the data processing and computation is hold by each sensor node without the need of sink nodes (e.g. central station). As the likelihood function of the LNSM is not convex with respect the unknown positions, a basic gradient descent method suffers from the initialization issue. We take inspiration from the two phase methods of $[14,15]$, which consider an initial guess step followed by a cooperative refinement step. In order to improve the accuracy achieved by the B-MLE [13], we use the on-line distributed stochastic approximation (DSA) approach of [16] to minimize the mean square error of the estimated distance derived from the noisy RSSI received data. The iterative DSA algorithm involves a first local update based on stochastic gradient descent (see [16]) given a new RSSI measurement at each sensor node. Then, an asynchronous communication step (see [17]) involves the exchange of information between two randomly selected nodes to reach a consensus on the set of estimated positions. It allows each sensor node to build a local map of itself and its neighbor nodes. Our approach is tested on real indoor scenarios: the two rooms from [18] and a number of selected sensor nodes from the FIT IoT-LAB Rennes platform [19] (platform previously known as SensLAB where the new generation of sensor nodes ARM Cortex M3 with ATMEL radio chipset will be gradually incorporated).

The paper is organized as follows. First, Section II gives an overview of the proposed concept. Section III introduces the notation in use throughout the paper and recalls the lognormal shadowing model (LNSM) for the observed signal. The main equations of the biased lognormal shadowing model (B-LNSM) are introduced in Section IV-A. The initialization phase of the localization algorithm proposed here is presented in Section IV-B, while the refinement phase is detailed in Section V. Section VI describes the test bed of our experimental results, which are subsequently summarized, compared and discussed. Finally, conclusions are provided in Section VII.

\section{WSN-Based Reindeer's Calf Identification Concept}

A technique based on wireless sensor networks to identify the ownership of the calves is presented in this work. It is proposed to furnish the new born calves with an electronic identification device, such as RFID tags, GPS collars or UHF nodes, with a system setup facilitated with receivers, gateways and necessary software. The reindeer herd is confined in a restricted space, which is equipped with a grid of signal receivers, corresponding to the transmitter devices attached to the reindeer, and using proper localization algorithms. Movement of transmitter tags is tracked in order to recognize the pairs of tags which keep close together most of the time, which indicate the mother and calf reindeer. Due to the fact that reindeer is a wild animal species, they tend to be more cautious than domestic animals; hence they tend to avoid confined spaces and close objects mounted by humans, which makes the use of passive RFIDs less efficient due to their low range, as the receivers have to be placed at a close proximity to the RFIDs. This demands the application of active RFIDs to both mothers and calves, or the use of other sensor network devices such as GPS collars or UHF nodes and respective gateways. For example, active RFID tags operate at higher frequencies than passive tags because of their on-board power source, commonly $455 \mathrm{MHz}, 2: 4 \mathrm{GHz}$, or $5: 8 \mathrm{GHz}$ - depending on application's read range and memory requirements. Readers can communicate with active RFID tags across 20 to 100 meters. In the same way, tags can track the movement of highly mobile objects in wider areas than passive tags [20]. In this procedure, the pen where the animals are to be gathered for tagging is facilitated with a WSN, assuming that the WSN is readily available with a set of low cost sensor nodes such those 
Citation: Morral G, Mohamad Y, Diengy NA (2017) Efficient Distributed Localization Algorithm for Ownership Identification of Reindeer Calf Using Wireless Sensor Networks. Int J Comput Softw Eng 2: 120. doi: https://doi.org/10.15344/2456-4451/2017/120

Page 2 of 7

used in Section VI (WSN430). Central sensor nodes are mounted on posts at the centers of the circles indicated in (Figure 1), which indicate the coverage range of the routers.

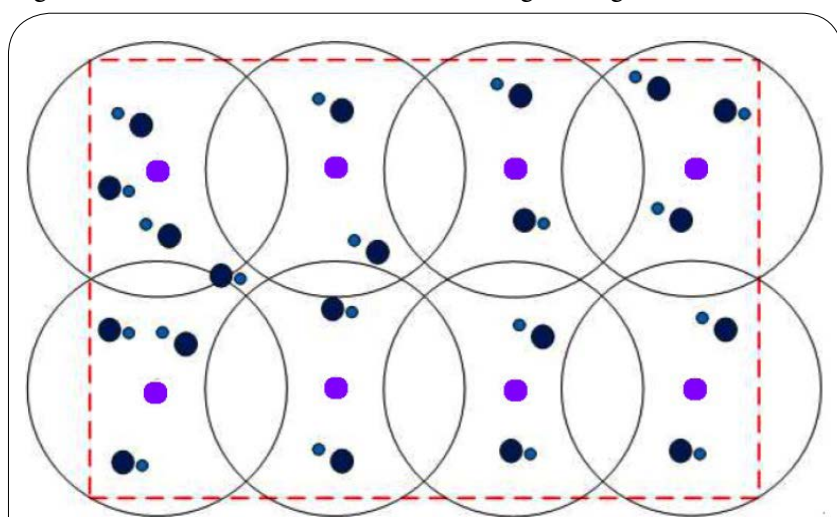

Figure 1: Proposed configuration of the localization set-up. Suggested area of the pen is $50 \times 90 \mathrm{~m}^{2}$ (dashed rectangle). Large black circles represent coverage area of the central sensor nodes which are represented by the violet dots. Mother and calf reindeer are represented by the large and small pairs of coloured dots in dark and light blue.

Coverage of the wireless network is configured in such a way to ensure exposure of the whole pen, with minor inevitable pockets of uncovered areas, which have to be kept to a minimum. RFID tags are tracked and location data is logged into a computer program, where data is analyzed to match couples of tags together.

\section{Framework}

The following framework to solve the localization problem in a distributed manner using WSNs, i.e. local measures and communications through the network of sensor nodes, is based on the previous work proposed in [21].

\section{Notation}

Consider the context of $\mathrm{N}$ sensor nodes placed within a twodimensional space $\mathrm{p} \times \mathrm{qm}^{2}$ whose unknown positions are defined by the set $\left\{Z_{i}\right\}_{\forall i}$ which is also represented by every pair of coordinates as $\left\{\left(x_{1}-y_{1}\right), \ldots,\left(x_{N}, y_{N}\right)\right\}$. The euclidean distance between each pair of nodes $\mathrm{i}$ and $\mathrm{j}$ is defined as $d_{i j}=\left|z_{i}-z_{j}\right|=\sqrt{\left(x_{i}-x_{j}\right)^{2}+\left(y_{i}-y_{j}\right)^{2}}$. The set $\left\{Z_{k}\right\}_{\forall k}=\left\{(a 1, b 1), \ldots,\left(a_{M}, b_{M}\right)\right\}$ defines the known positions from $\mathrm{M}$ landmarks, or anchor nodes, with $\mathrm{M} \ll \mathrm{N}$. Given a radio range $\mathrm{r}$, we define for each node $i$ the set of its neighboring nodes as $N_{i}=\left\{\forall{ }_{j} \mid d_{i j}<r\right\}$ and the set of its neighboring landmarks as $M_{i}=\left\{\forall k \mid d_{i k}<r\right\} \quad$. The objective of each sensor node is to localize itself and obtain a local map from its estimate position and those from its neighboring sensor nodes. The abbreviation r.v. stands for random variable while the abbreviation i.i.d stands for independent and identically distributed.

\section{Benchmark: log-normal shadowing model (LNSM)}

We recall the empirical model used to describe the received signal strength indicator (RSSI) data as a function of the distances between the sensor nodes. The log-normal shadowing model (LNSM) is based on the log-distance path loss model of [8], which describes the average path loss $\mathrm{PL}(d)$ expressed in $\mathrm{dB}$ for a distance $d$ as given a path loss exponent $\eta$ depending on the propagation medium, a reference distance $\mathrm{d}_{\mathrm{o}}$ and its corresponding path loss $\operatorname{PL}\left(d_{0}\right)$ value. The LNSM captures the random shadowing effects that may occur at different locations having the same distance separation, i.e., the addition of a zero mean Gaussian r.v. (random variable) $\varepsilon \sim \mathrm{N}\left(0, \sigma^{2}\right)$ to the average path loss $\operatorname{PL}(d)$. The RSSI can be described as the noisy received power at distance $d$ given an average path loss $\mathrm{PL}(d)$, and an emitted power $\mathrm{P}_{\mathrm{T}}$ i.e., $p(d)=P T-P L(d)+\varepsilon$. Assume that $\mathrm{d}_{0}=1 \mathrm{~m}$ as usual in indoor context, the emission power $\mathrm{P}_{\mathrm{T}}$ is $0 \mathrm{dBm}$ and define $\mathrm{PL}\left(\mathrm{d}_{0}\right)$ as a parameter $\mathrm{P}_{0}$. The general expression of the LNSM for the RSSI r.v. P is: $P=-P_{0}-\eta 10 \log _{10} d+\varepsilon \sim N\left(-P_{0}-\eta 10 \log _{10} d, \sigma^{2}\right)$ (1)

The above equation (1) depends on the propagation parameters $\mathrm{P}_{0}, \eta$ and $\sigma^{2}$, which may not be homogeneous in indoor scenarios. A maximum likelihood estimator (MLE) can be used to learn these parameters from the RSSI values collected from a set of landmarks whose positions are known. Since the average values of the RSSI measured at different positions do not always decrease with the distance (see the learning phase in [10]), we let the parameters of the propagation model differ from one landmark to another. As a result,
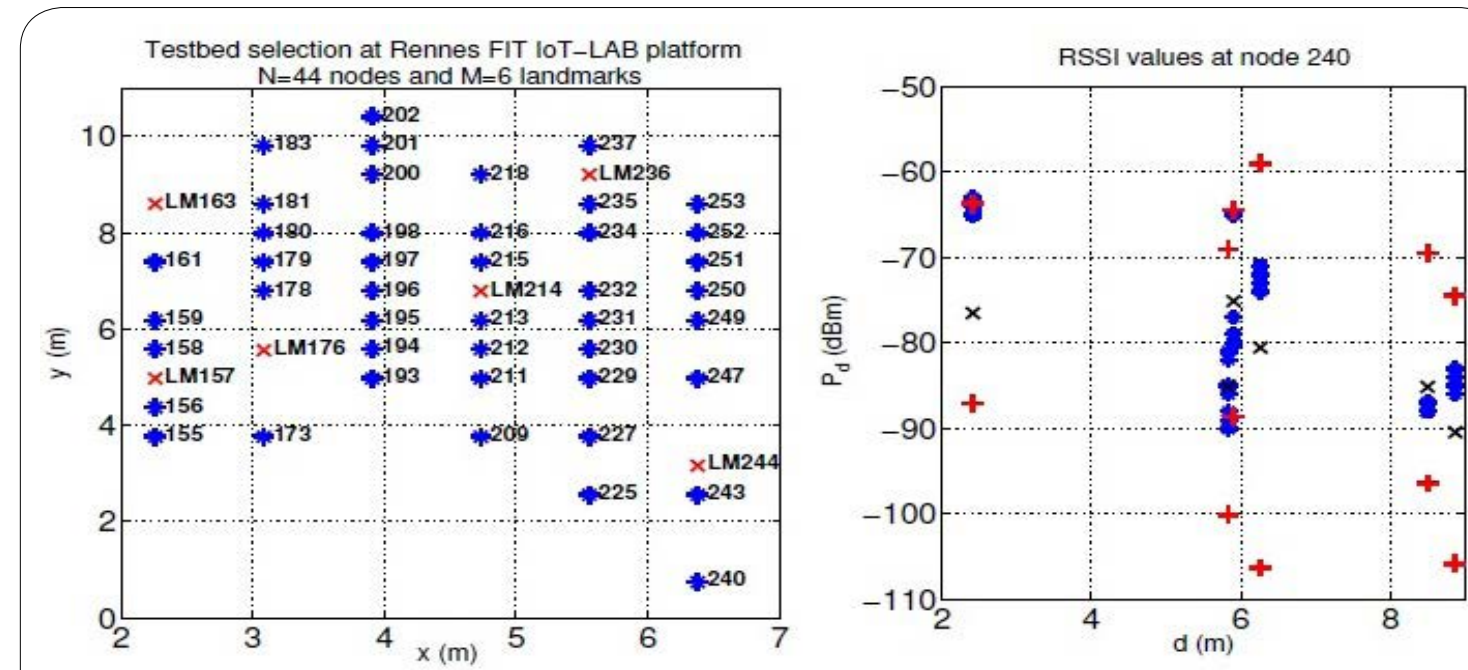

Figure 2. Testbed at FIT IoT-LAB platform (left) and RSSI values (right) collected at node 158 and node 183 from the 6 landmarks. The marker $\left({ }^{*}\right)$ highlights the real RSSI values. The markers (5) and (+) indicate, respectively, the average and the minimum and maximum values from 100 i.i.d. random samples drawn when considering the theoretical LNSM in (1) given the estimated parameters in Table 1. 
Citation: Morral G, Mohamad Y, Diengy NA (2017) Efficient Distributed Localization Algorithm for Ownership Identification of Reindeer Calf Using Wireless Sensor Networks. Int J Comput Softw Eng 2: 120. doi: https://doi.org/10.15344/2456-4451/2017/120

Page 4 of 7

we do not treat all landmarks equally during the statistical estimation (cf. Tables ?? and 1). Moreover, in order to show how, in general, the definition (1) does not match in a real indoor environment, (Figures 2) highlights the presence of a bias probably due to multipaths. For instance, in Figure 2 node 240 collects $(27 ; 7 ; 21 ; 203 ; 26 ; 533)$ packets from landmarks whose identifiers (Node_ID) are (157; 163; $176 ; 214 ; 236 ; 244)$. There is a bias in values coming from landmark 244 , even if this is the one closest to node 240 . This sensor node is placed at the boundary and immediately close to the wall of the room.

Landmarks 163 and 176, which are placed at distances 8:87mand $5: 83 \mathrm{~m}$ from node 240 , are aligned with its line-of-sight. Thus, 240 receives the smallest number of packets ( 7 and 21 , respectively) from these nodes, and the RSSI values slightly match with the model.

\section{Position Estimation: Intialization}

\section{Principle: biased log-normal shadowing model}

This section recalls the dynamic method introduced by [13] to estimate the position of a sensor node from a set of landmarks. The sensor node seeks to reduce the effect of any potentially aberrant landmark whose measurements do not improve localization accuracy. This effect is compensate by introducing a constant bias which becomes an additional variable to estimate and replaces the lognormal shadowing model of the measurements associated to this landmark. Let us denote by RSSI th $_{\mathrm{L}}$ the $\mathrm{j}^{\text {th }}$ RSSI sample measured by the sensor node on packets coming from a given landmark $\mathrm{L}$. If we denote by $\mathrm{P}_{0, \mathrm{~L}, \mathrm{~L}}$ and $\sigma_{\mathrm{L}}{ }^{2}$ the LNSM specific parameters for landmark L, we can rewrite the equation (1) in the general case, replacing measurements coming from a given landmark $\mathrm{O}$ by a constant bias, $\beta$, as follows:

$$
R S S I_{j, L}=-\left(P_{o, L}+10 \eta_{L} \log _{10} d_{L}\right) 1_{L \neq \mathrm{o}}+\beta 1_{L=o}+N\left(0, \sigma_{L}^{2}\right),
$$

where $\mathrm{dL}$ is the distance to landmark $\mathrm{L}$ that we try to estimate and 1 is the indicator function, which is equal to 1 when the subscript expression is true and 0 otherwise. Abnormal landmarks can be detected from equation (2), and the biased LNSM can be fully characterized. Thus, the biasedmaximum likelihood estimator may be used to compute the sensor node position from RSSI measurements collected from $\mathrm{M}$ landmarks. The aberrant landmark can be identified by comparing the global likelihood values when each landmark is considered as outlier.

\section{Biased-maximum likelihood location estimation method (B-MLE)}

Combining all the measured values altogether, we can apply a maximum likelihood estimator on this new model to compute the likelihood expressions in the case where landmark $\mathrm{O}$ is considered as abnormal. If we denote by TL the number of samples received from landmark $\mathrm{L}$, the likelihood function is expressed as follows for every landmark $\mathrm{L} \neq \mathrm{O}$ :

$$
\begin{aligned}
& \text { landmark } \mathrm{L} \neq \mathrm{O} \text { : } \\
& L_{L}(x, y)=-T_{L} \log \sigma_{L}^{2}-\frac{1}{2} \sum_{j=1}^{T_{L}}\left(\frac{R S S I_{j, L}+P_{0, L}+10 \eta \log _{10} d_{L}}{\sigma_{L}}\right)^{2}
\end{aligned}
$$

and for the outlier (abnormal) landmark, $\mathrm{O}$, it becomes:

$$
L_{o}(\beta)=-T_{o} \log \sigma_{o}^{2}-\frac{1}{2} \sum_{j=1}^{T_{O}}\left(\frac{R S S I_{j, O}-\beta}{\sigma_{O}}\right)
$$

The global likelihood function from the data set, reflecting the coherence of the whole system when landmark $\mathrm{O}$ is the outlier one is then simply the sum of equations (3) and (4) over the M landmarks:

$$
L_{O}(x, y, \beta)=\sum_{L=1, L \neq O}^{M} L_{L}(x, y)+L_{o}(\beta)
$$

Thus, the maximum likelihood criterion applied to equation (5) is used to infer the sensor node position and the bias. Using the fact that it can be solved by separating the problem, the B-MLE solution is given by:

$$
\begin{aligned}
& (x o, y o, \beta o)=\max _{x, y} \sum_{L=1, L \neq 0}^{M} L_{L}(x, y)+\max _{\beta} L_{O}(\beta) \\
& \text { Where } \beta_{O}=\frac{1}{T_{O}} \sum_{j=1}^{T_{O}} R S S I_{j, O}
\end{aligned}
$$

\section{Position Refinement: Improving The Accuracy}

\section{Principle: distributed stochastic approximation (DSA)}

The objective in this phase is described under a global maximum likelihood estimation of the log-distances between the $\mathrm{N}$ sensor nodes positions. From the LNSM in equation (1), we define the estimated log-distance $\hat{D}$ given the estimated average RSSI $\bar{P}($ i.e, from a collection of T i.i.d. noisy RSSI measurements $(\mathrm{P} 1, \ldots, \mathrm{PT})$ at distance $\mathrm{d}$ compute the estimated average $\left.\bar{P}=\frac{1}{T} \sum_{t=1}^{T} P_{t}\right)$ as follows:

$$
\hat{D}=-\frac{\bar{P}+P_{0}}{10 \eta}=\log _{10} d+\frac{\varepsilon}{10 \varepsilon} \sim N\left(\log _{10} d, \frac{\sigma^{2}}{100 T^{2} \eta^{2}}\right)
$$

We define the sets of RSSI measurements collected for each sensor node i as $\left\{\bar{P}_{i j}\right\} \forall k \in N_{i}$ from its neighboring sensor nodes and $\left\{\bar{P}_{i k}\right\} \forall k \in M_{i}$ from its neighboring landmarks. For each sensor node i, the set of positions of the neighboring nodes' is denoted as $\left(x^{(i)}, y^{(i)}\right)=\left\{\left(x_{j}, y_{j}\right)\right\} \forall j \in N_{i}$. The aim is to solve the global optimization problem defined as:

$\min _{(x, y)} \sum_{i=1}^{N} f_{i}\left(x^{(i)}, y^{(i)}\right)$

Where $f_{i}\left(x^{(i)}, y^{(i)}\right)=\sum_{j \in N_{i}}\left(\hat{D}_{i, j}-\log _{10}\left|Z_{i}-Z_{i}\right|\right)^{2}+\sum_{k \in M_{i}}\left(\hat{D}_{i, k}-\log _{10}\left|Z_{i}-Z_{k}\right|\right)^{2}$

On-line gossip-based implementation

Problem (8) is solved by means of the on-line distributed stochastic approximation algorithm (DSA). At any time $t \geq 0$, each sensor node $i$ updates the sequence of its estimated neighboring nodes' positions $\left(x_{t}^{(i)}, y_{t}^{(i)}\right)=\left\{\left(x_{j, t}^{(i)}, y_{j, t}^{(i)}\right)\right\} \forall j \in N_{i} \quad$ corresponding to its local map. The iterative procedure of the DSA algorithm relies on the following two steps:

[Local gradient descent step][16] At iteration t each sensor node i computes a temporary estimate of its positions' set from the local current measurements $\left\{P_{i j}^{t}\right\} \forall j \in N_{i}$ and $\left\{P_{i j}^{t}\right\} \forall k \in M_{i}$ as:

$\sim(i) \sim(i)$

$\left(\tilde{x_{t}}, \tilde{y}_{t}\right)=\left(x_{t-1}^{(i)}, y_{t-1}^{(i)}\right)-\gamma t \nabla f_{i}\left(x_{t-1}^{(i)}, y_{t-1}^{(i)}\right)$

Where $(\gamma t)_{t} \geq 1$ is a decreasing step sequence s.t. $\gamma t=1 / \sqrt{t}$

[Gossip averaging step] [17] At each iteration time $t$ two uniformly random selected nodes exchange their common estimated positions and average their values. The final updates are set as follows:

$$
\begin{aligned}
& \left(x_{l, t}^{(i)}, y_{l, t}^{(i)}\right)=\frac{\left(\tilde{x}_{l, t}^{(i)}, \tilde{y}_{l, t}^{(i)}\right)}{2}+\frac{{\tilde{\left(x_{l, t}^{(j)}\right.}, \tilde{y}_{l, t}^{(j)}}^{(j)}}{2} \\
& \left(x_{l, t}^{(j)}, y_{l, t}^{(j)}\right)=\left(x_{l, t}^{(i)}, y_{l, t}^{(i)}\right), \quad \forall l \in N_{i} \cap N_{j},
\end{aligned}
$$

Otherwise $\forall l \notin N_{i} \cap N_{j}$ and $\forall m \neq i, j$ then,

$$
\left(x_{l, t}^{(m)}, y_{l, t}^{(m)}\right)=\left(\tilde{x}_{l, t}^{(m)}, \tilde{y}_{l, t}^{(m)}\right) \text {. }
$$


Citation: Morral G, Mohamad Y, Diengy NA (2017) Efficient Distributed Localization Algorithm for Ownership Identification of Reindeer Calf Using Wireless Sensor Networks. Int J Comput Softw Eng 2: 120. doi: https://doi.org/10.15344/2456-4451/2017/120

Page 5 of 7

\section{Numerical Results: Experiences From WSNS}

For the considered testbeds, the procedure is described as follows. Each of the M landmarks broadcasted 100 frames. Then, the first $\mathrm{N}_{\mathrm{L}}$ sensor nodes selected for the learning phase computed the set of the propagation model parameters $\left\{\left(P_{0, k, \eta k}, \sigma_{k}^{2}\right)\right\}_{k}^{M}=1$ as detailed in [10]. We chose two different sizes NL of the learning data set, a small set involving the first 10 sensor nodes' positions and a large one from the first 25 sensor nodes' positions. The RSSI values were collected from the set of received frames, and then the parameters were estimated by applying the maximum likelihood criterion. The remaining N-NL sensor nodes were able to compute a first estimate of their positions using the B-MLE [6] given the parameters and the RSSI values from theirs corresponding received frames. The refinement phase was subsequently applied assuming the latter positions as the initialization values as detailed by our algorithm in Section V-B. At each iteration, a single frame was broadcasted by each sensor in order to compute the local estimates defined by equation (9) and only two sensors randomly selected exchanged their common estimate positions which were finally updated as the average given by equation (10).

\section{Testbed description: FIT IoT-LAB platform}

The testbeds were chosen at the FIT IoT-LAB's platform of size $5 \times 9 \mathrm{~m}^{2}$ involving the positions of 44 sensor nodes and 6 landmarks which are located in a big storage room of size $8 \times 11 \mathrm{~m}^{2}$ containing different objects. Sensor nodes are placed at the ceil which is $1: 9 \mathrm{~m}$ height from the floor in a grid organization. There was no one in the room most of the time and there was only a wireless access point located in the corridor which is separated by a cinder wall (no electromagnetically isolating). The estimated parameters are summarized in Table 1.

\begin{tabular}{|l|l|l|l|l|l|l|}
\hline LM_ID & LM157 & LM163 & LM176 & LM214 & LM236 & LM244 \\
\hline P0 & 62019 & 63.61 & 58.4 & 63.33 & 58.55 & 67.67 \\
\hline$\eta$ & 1.76 & 2.83 & 3.39 & 1.98 & 2.80 & 2.29 \\
\hline$\sigma 2$ & 19.06 & 40.87 & 37.04 & 75.62 & 30.03 & 18.97 \\
\hline
\end{tabular}

Table 1. Estimated parameters from the FIT IoT-LAB testbed when the small data set of 10 sensor nodes is selected.

\section{Comparison and discussion}

We have tested the algorithms proposed in Sections IV-B and V-B whose respective procedures are defined by equations (6) and (9)(10). In order to evaluate the performance of such methods and quantify the achieved accuracy, we define the normalized mean deviation (NMD) as the average mean deviation (MD) error of the $\mathrm{N}$ estimated positions normalized by the testbed dimensions i.e. $\mathrm{p} \times \mathrm{qm}^{2}$. It can be defined as: $\mathrm{NMD}=\frac{1}{N} \sum_{i=1}^{N} \mathrm{NMD}_{i}=\frac{1}{\sqrt{P^{2}+q^{2}}} \frac{1}{N} \sum_{i=1}^{N}\left\|\tilde{Z}_{i}-Z_{i}\right\|$

Where $\left\{z_{i}\right\}_{\forall i}$ are the set of the $\mathrm{N}$ estimated positions. Figure 3 illustrates the decreasing curves of the average mean deviation of the $\mathrm{N}$ positions along the iteration instant $\mathrm{t}$ from each testbed, i.e. Experience 1, Experience 2 and Experience 3. Note that as being a real environment, the algorithm converged to an asymptotic error, which may depend on the testbed's parameters.

Figure 3 shows the evolution of the mean deviation error MD as a function of the iterative steps during the refinement phase, which involves the communication between two sensor nodes at each step. The earliest improvement ( 24 steps) has been obtained in Experience

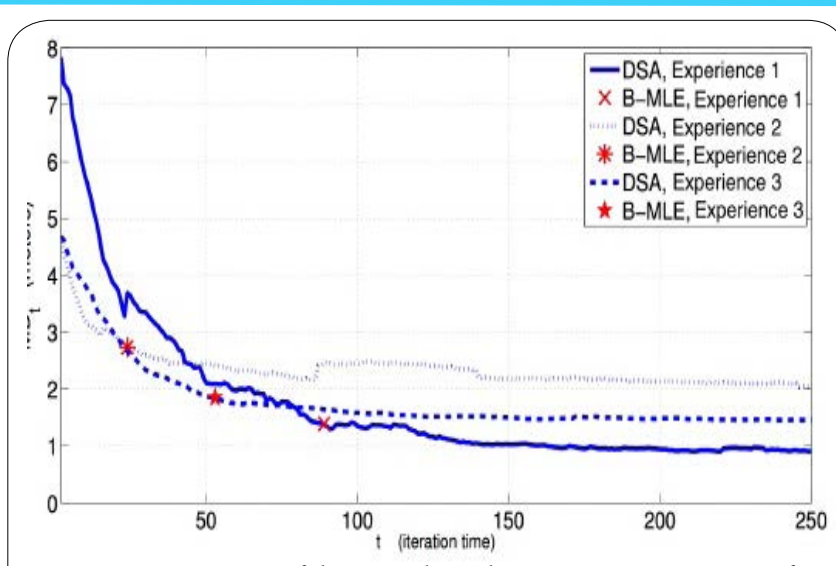

Figure 3. Convergence of the DSA along the iteration time instants $t$ for each testbed when considering the small data learning set $(\mathrm{NL}=10)$. Markers $(x, *, \quad)$ emphasize the iteration time when the error MD of the DSA refinement phase is lower than the error MD computed after the position estimation phase by the B-MLE.

2 of the testbed. However, for this experience the accuracy after the refinement phase has been the worst achieved as the curve of the mean deviation error remains always above. The best accuracy was achieved at Experience 1 for the considered testbed even if the convergence was slower and 89 refinements steps were required to improve the mean deviation error. As reported in Table 2, less than $80 \mathrm{~cm}$ was obtained from Experience 1 of the testbed.

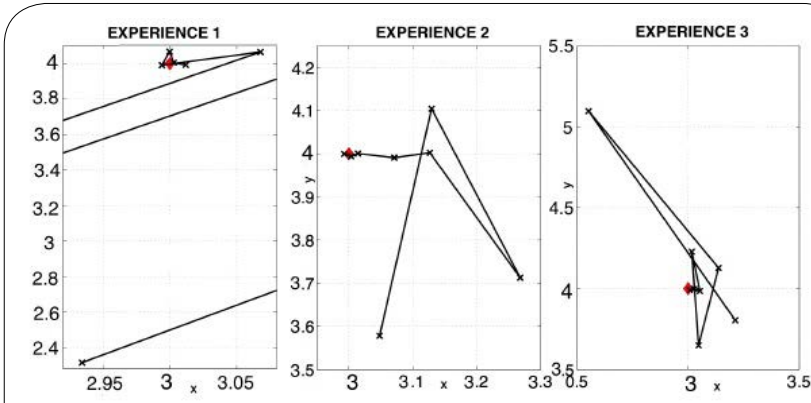

Figure 4. Convergence of the estimated position node whose id is 173 during the refinement phase through algorithm DSA at different iteration times $(t=0,10,20,30,50,100,200,500)$.

Figure 5, 6 and 7 show the corresponding normalized NMD values of each sensor node position $\left\{N M D_{i}\right\}_{\forall i}$ before and after the refinement phase. For Experience 1s about $76 \%$ positions were improved when the small learning data set was chosen $(\mathrm{NL}=10)$. However, the percentage decreased to $55 \%$ when 25 positions where considered during the learning phase.

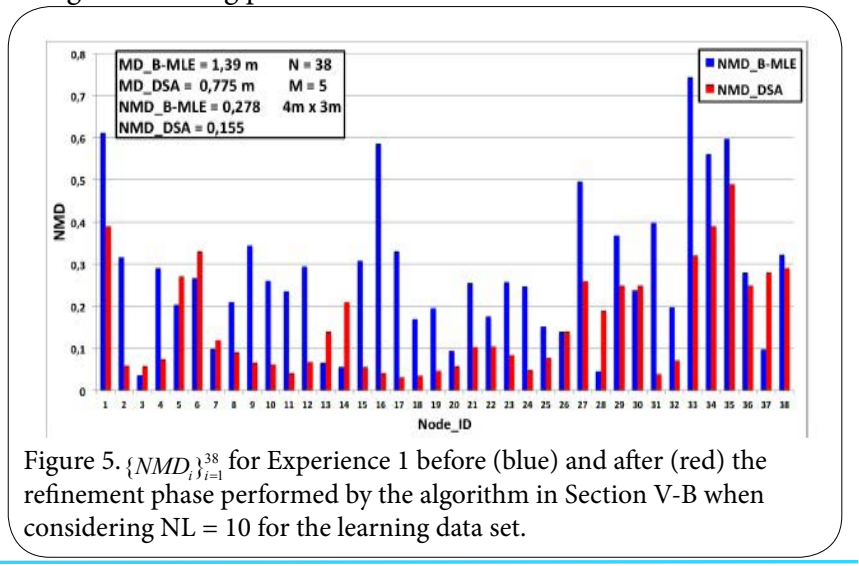




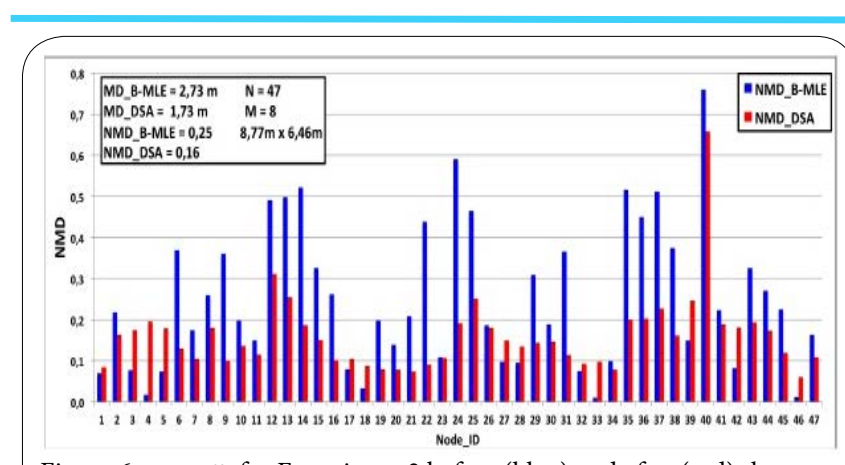

Figure 6. $\left\{N M D_{i}\right\}_{i=1}^{38}$ for Experience 2 before (blue) and after (red) the refinement phase performed by the algorithm in Section V-B.

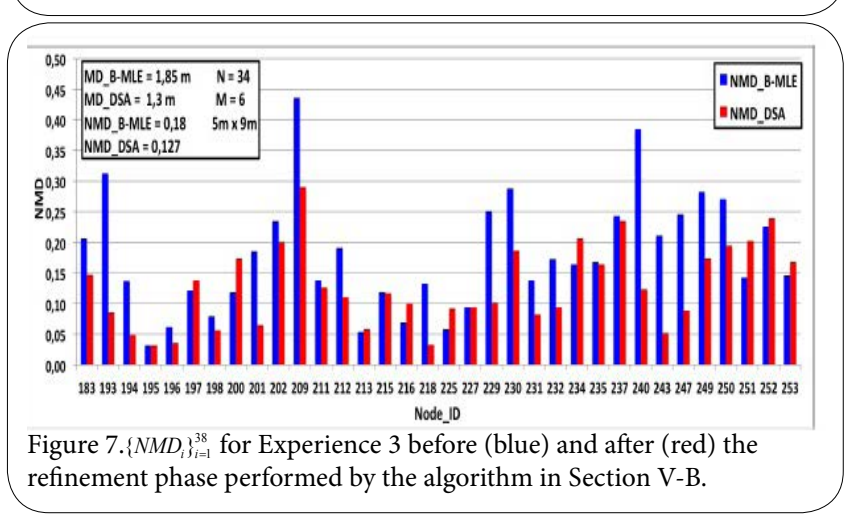

In order to summarized the results for each testbed, Table 2 displays the average error, both MD in meters and the respective normalized value as NMD. The numerical results are reported when considering the two sizes of data sets chosen during the learning phase of the B-MLE $\left(N_{L}=10\right.$ and $\left.N_{L}=25\right)$. In addition, we computed the ratio of the accuracy improvement as the percentage $(1-\rho) \%$ where $\rho$ defines the ratio between the NMD achieved after the refinement phase with the proposed DSA (Section V-B) and those achieved by the B-MLE. We also computed the ratio regarding the number of improved positions after the refinement phase (see the ratio Positions improved in Table 2). From the results reported in Table 2, we are led to conclude that, in general, the best accuracy improvement has been obtained in the case of the small data learning set, i.e., $\mathrm{NL}=10$. The best accuracy about $70 \mathrm{~cm}$ is achieved from Experience 1. However, the latter case required the highest number of pairwise communications between the sensor nodes during the refinement phase. Our results are consistent compared to other experiments involving real indoor scenarios with similar dimensions and number of sensor nodes. See for instance the accuracy between 1:5-2:5 $\mathrm{m}$ from the experiment of [9] or the $2: 27 \mathrm{~m}$ reported in [22].

\section{Conclusion}

In this paper, a novel application of WSN based on RFID tags is addressed for ownership identification of new-born reindeer calves, in order to mark the calves with ear marks or identification tags of their relevant owners. The principle of identification is based on matching reindeer calves to their mothers through estimating their locations in the herd, while finding a proximity between a reindeer cow and her calf. In the proposed set-up, active RFID ear tags, which are fitted to the animals, are utilized as nodes. Such tags normally have an accuracy of 3 - 5 meters when determining the location, and when used with central nodes (gateways), it is possible to allow a large number of tags to communicate simultaneously with a single access point without choking the wireless network. The traditional RFID technology would have required a larger number of readers, in order to determine the location of a single tag, with the same precision as the wireless network. For the proposed application, the system needs the implementation of a localization algorithm. Various localization techniques relying on distance measurements in WSNs (related to RSSI magnitude) have been considered and thoroughly discussed in this paper. Several existing techniques has been investigated to estimate locations of target tags considering range measurements, which can be employed in proper computer algorithms to analyze the gathered data-base of animal tracking. Among all possibilities, RSSI is considered here as the most economic and practical choice for the proposed application. Since the accuracy of the estimated positions is not the priority, we proposed a more flexible and cheaper solution thanks to a distributed algorithm. The implemented algorithm is based on local measurements and communications through neighboring nodes. In particular, we considered two phases including an initialization MLE phase and a refinement phase based on a DSA algorithm. The latter enables each sensor node to track its own position and those from its neighbors. This leads the process of matching a reindeer calf to its mother in a robust way. The advocated

\begin{tabular}{|c|c|c|c|c|c|c|}
\hline & \multicolumn{2}{|c|}{ Experience 1 } & \multicolumn{2}{c|}{ Experience 2 } & \multicolumn{2}{c|}{ Experience 3 } \\
\hline Testbed & B-MLE & DSA & B-MLE & DSA & B-MLE & DSA \\
\hline Method & 1.39 & 0.77 & 2.73 & 1.73 & 1.85 & 1.3 \\
\hline NMD & 0.28 & 0.16 & 0.25 & 0.16 & 0.18 & 0.13 \\
\hline Improvement (\%) & 44.3 & 36.7 & 29.5 & & & \\
\hline Positions improved(\%) & 76 & 72 & 74 & & & \\
\hline
\end{tabular}

\begin{tabular}{|c|c|c|c|c|c|c|}
\hline \multirow{2}{*}{$\begin{array}{l}\text { Testbed } \\
\text { Method }\end{array}$} & \multicolumn{2}{|c|}{ Experience 1} & \multicolumn{2}{|c|}{ Experience 2} & \multicolumn{2}{|c|}{ Experience 3} \\
\hline & B-MLE & DSA & B-MLE & DSA & B-MLE & DSA \\
\hline $\mathrm{MD}(\mathrm{m})$ & 1.35 & 1.31 & 2.27 & 1.64 & 2.27 & 1.84 \\
\hline NMD & 0.27 & 0.26 & 0.22 & 0.15 & 0.22 & 0.18 \\
\hline Improvement(\%) & 3.8 & 30.5 & 19 & & & \\
\hline Positions improved(\%) & 55 & 63 & 68 & & & \\
\hline
\end{tabular}

Table 2. Localization error averaged over the $\mathrm{N}$ estimated sensor nodes' positions for each of the three considered testbeds when using the small data set of 10 positions (up) and the big data set of 25 (down). 
Citation: Morral G, Mohamad Y, Diengy NA (2017) Efficient Distributed Localization Algorithm for Ownership Identification of Reindeer Calf Using Wireless Sensor Networks. Int J Comput Softw Eng 2: 120. doi: https://doi.org/10.15344/2456-4451/2017/120

Page 7 of 7

method suffers from a few limitations pertaining to the number of tags that can be covered using one central node reader and coverage of the pen area, where the reindeer herd is gathered for identification, which could create further problems pertaining to transmission, repeatability and accuracy. The proposed method reduces the amount of effort and time in performing the identification process and paves the way for the implementation of wireless sensor network enabled RFID tags for animal welfare.

\section{Conflict of Interest}

No authors have a conflict of interest or any financial tie to disclose.

\section{References}

1. The international centre for reindeer husbandry (icr), reindeer herding, 2015. [online] available.

2. Paine R (1994) Herds of the Tundra: A portrait of Sami Reindeer Pastoralism. Smithsonian Institution Press.

3. TyleraJ NJC, Turi JM, SundsetK MA, Strøm Bulld K, Sara MN, et al. (2007) Saami reindeer pastoralism under climate change: Applying a generalized framework for vulnerability studies to a sub-arctic social-ecological system. Global Environmental Change 17: 191-206.

4. Mustafa M, Hansen I, Eilertsen S (2013) Animal Sensor Networks: Animal Welfare under Arctic Conditions. In SENSORCOMM 2013: The Seventh International Conference on Sensor Technologies ans Application, Barcelona (Spain).

5. Langendoen K, Reijers N (2003) Distributed localization in wireless sensor networks: a quantitative comparison. Computer Networks 43: 499-518.

6. Patwari N, Ash JN, Kyperountas S, Hero AO, Moses RL, et al. (2005) Locating the Nodes : cooperative localization in wireless sensor networks. IEEE Signal Processing Magazine, 22: 54-69.

7. Mao G, Fidan B, Anderson B (2007) Wireless sensor network localization techniques. Computer Networks 51: 2529-2553.

8. Rappaport TS (1996) Wireless Communications: Principles and Practice. Prentice Hall.

9. Sugano M, Kawazoe T, Ohta Y, Murata M (2006) Indoor localization system using rssi measurement of wireless sensor network based on zigbee standard. In Wireless and Optical Communications, pages 1-6. IASTED/ ACTA Press.

10. Dieng N A, Charbit M, Chaudet C, Toutain L, Meriem TB, et al. (2012) Experiments on the RSSI as a Range Estimation for Indoor Localization. In (NTMS), pages 1-5.

11. Bahl P, Padmanabhan VN (2000) RADAR: An In-Building RF-Based User Location and Tracking System. In INFOCOM, pages 775-784.

12. Elnahrawy E, Xiaoyan L, Martin RP (2004) The limits of localization using signal strength: a comparative study. In In First Annual IEEE Conference on Sensor and Ad-hoc Communications and Networks, pages 406-414.

13. Dieng NA, Charbit M, Chaudet C, Toutain L, Meriem TB et al. (2012) A Multi-Path Data Exclusion Model for RSSI-based Indoor Localization. In 15th International Symposium on Wireless Personal Multimedia Communications (WPMC), pages 336-340.

14. Savarese C, Rabaey J, Langendoen K (2002) Robust positioning algorithm for distributed ad-hoc wireless sensor network. In USENIX Technical Annual Conference, Monterey.

15. Savvides A, Park H, Srivastava MB (2002) The Bits and Flops of the N-hop Multilateration Primitive For Node Localization Problems. In Proceedings of the 1st ACM International Workshop on Wireless Sensor Networks and Applications, WSNA '02, pages 112-121. ACM.

16. Bianchi P, Fort G, Hachem W (2013) Performance of a Distributed Stochastic Approximation Algorithm. IEEE Transactions on Information Theory 59: $7405-7418$.

17. Boyd S, Ghosh A, Prabhakar B, Shah D (2006) Randomized Gossip Algorithms. IEEE Transactions on Inform. Theory 52: 2508-2530.

18. Dieng NA, Charbit M, Chaudet C, Toutain L, Meriem TB, et al. (2013) Indoor Localization in Wireless Networks based on a Two-modes Gaussian Mixture Model. In IEEE 78th Vehicular Technology Conference (VTC Fall), pages $1-5$.

Int J Comput Softw Eng

ISSN: $2456-4451$
19. Fit iot-lab : very large scale open wireless sensor network testbed.

20. Mustafa M, Hansen M, Eilertsen S, Pettersen E, Kronen A, et al.(2013) Matching Mother and Calf Reindeer Using Wireless Sensor Networks. In 5th International Conference on Computer Science and Information Technology (CSIT 2013), Amman (Jordan).

21. Morral G, Dieng NA (2014) Cooperative RSSI-based Indoor Localization: B-MLE and Distributed Stochastic Approximation. In IEEE 80th International Vehicular Technology Conference (VTC2014-Fall), Santa Clara (USA)

22. Costa JA, Patwari N, Hero AO (2006) Distributed WeightedMultidimensional Scaling for Node Localization in Sensor Networks. ACM Transactions on Sensor Networks 2: 39-64. 